\section{Vas Narasimhan}

Novartis has about 500 ongoing clinical trials at any given time, at a huge expense. Since stepping into the role of global head of development at Novartis in 2014, Vas Narasimhan has been working to increase the efficiency and reduce the cost of these trials. He told Asher Mullard about his plans to overhaul the operational execution of drug development and integrate digital technologies into trials at Novartis.

\section{Q. What are your plans for development at Novartis?}

When I came in I saw an organization that had been successful and that had great people, but that needed to be re-envisioned to get to the next level of drug development. I have tried to focus the organization around two big themes: how can we deliver new medicines, and how can we rethink the science of operations and how we actually do drug development and execute trials?

On the medicine side, we looked at our mid-stage pipeline and asked which of these projects could have the biggest impact on health care and provide the biggest improvements versus standard of care? We deprioritized on the order of 20 different projects - in hepatitis antivirals, diabetes and metabolic disease - so that we could focus our resources and invest in areas where we can make bolder bets. Neuroscience is a big area where we can invest, and over the summer we did three different deals to build up our neuroscience pipeline. We are also looking at regenerative medicines and liver diseases where we think we can have a bigger impact.

I'm also really excited about the science of operations. A lot of our work comes down to whether we can execute clinical trials in a fast and efficient way. I personally believe that our industry has not invested enough in how we can transform and rethink how we operate. We have done some big data analyses on our historical performance over the past 10 years to find the fundamental drivers of cost and productivity in clinical trials. We learned some interesting insights in terms of how we select sites for our clinical trials, how we structure teams and how we design protocols to speed up and improve the efficiency of our trials. For example, we found that if we design studies with a mind to operational execution, even if it is just the inclusion-exclusion criteria for the right target patient population, we can dramatically affect how efficiently and effectively they are run. I also want to build datasets that allow us to evaluate on an ongoing basis how a trial is running, so that we can adapt the sites we are working with accordingly. We do adaptive design at the big level: the question here is whether we can, on a more micro level, adapt it to hit enrolment goals. You'd be surprised at how many non-performing trial sites don't enrol any patients at all.

Another area I would highlight is how much we can learn about the quality of our trials on an ongoing basis by really careful monitoring and by trying to create predictive algorithms that can look at a set of factors in a trial and warn us if quality might need to be addressed. We've identified close to 50 factors that can affect the quality of a trial site, and they are very straightforward things like the speed of data entry at the site. The magic is in trying to integrate all those different factors to develop algorithms that can predict which of our sites might have quality problems.

Drug development is fundamentally a people-driven enterprise and I also believe we need to invest far more in talent development and building better leaders. We need leaders who are not only great scientists, but who are also great people leaders.

Prior to this role, you spent most of your career at Novartis working on vaccines. How has this background affected your vision for pharmaceutical development? Vaccine development is a very different world, with a focus on public health, and full of intense interactions with a broad range of stakeholders. When I think about how this will help me in my new role, a couple of things come to mind.

First, I gained a strong sense of how people assess value for society. Cost-effectiveness analyses have been standard in vaccines for decades, and are the primary approach used for deciding whether vaccines are given broadly to cohorts of children and adults. I come with a strong sense of how payers

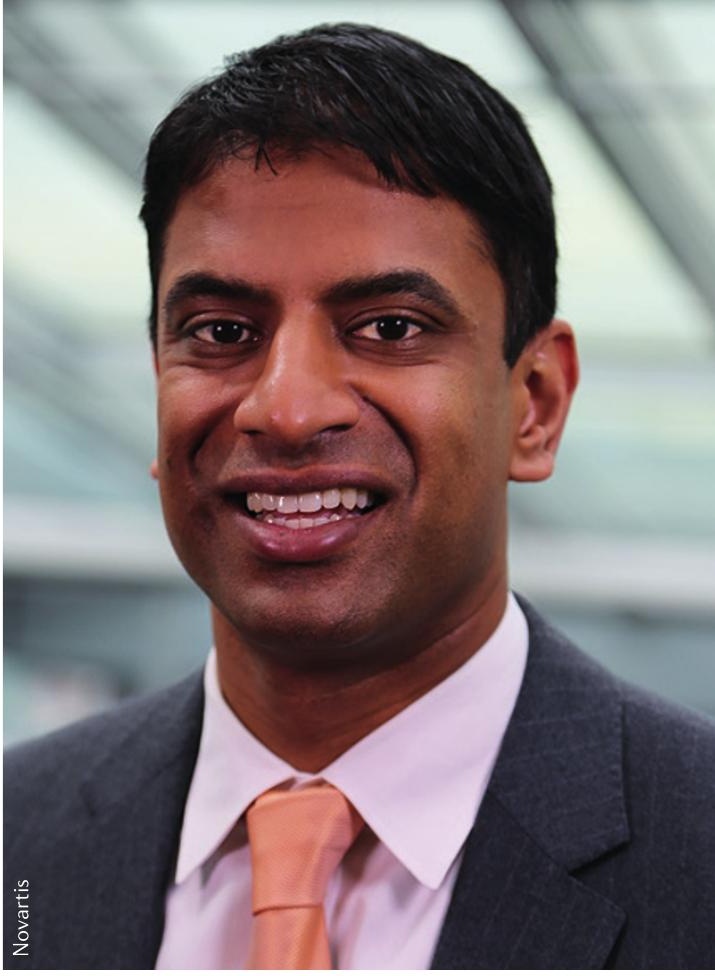

and health-care systems think about whether a medication will add value to the health-care system.

Second, I learned a lot about risk-benefit balance. In vaccines, especially when working on developing vaccines for children, there is a really high safety bar. Regulators have almost zero tolerance for risk with vaccines, and we have to generate a significant amount of benefit to vaccinate otherwise healthy people. In pharmaceuticals the risk-benefit equation is very different. I think having seen one extreme will help me understand how to strike the balance with our development-phase compounds.

Third, a lot of the science of operations comes from the fact that clinical trials of vaccines enrol tens of thousands of healthy people very quickly, sometimes in a matter of weeks or months. To do this, you have to be outstanding at operational execution. I think that if I can bring that to other areas of pharmaceutical development, the returns will be significant.

Q What challenges have you faced in moving from vaccines to pharmaceuticals?

I've had to go up a steep learning curve in really understanding the diversity of the different end points. In vaccines it is relatively straightforward: it is either a hard end point like onset of disease, or an immune response. Here we have a great diversity of end points. But, that said, the clinical pharmacology principles of drug development are generally similar across disease areas. I've also learned to rely on a great team, and at Novartis we work hard to build and develop the best scientists that we can, so I can rely on them to really know the details. 
Q. In 2014 Novartis sold most of its vaccines to GlaxoSmithKline. Does your move into this development role mean that Novartis might be interested in getting back into vaccines?

These events are completely unrelated. Our focus is on developing novel pharmaceuticals. My move was about finding someone who can lead an organization and rejuvenate how we do drug development.

Q. You've championed the use of digital technologies to improve clinical trials. How do you see this playing out?

Digital technologies will eventually transform how we do drug development. It is really a question of which company can scale these technologies first. On the development side, I see three ways that this will transform how we operate.

The first is just in terms of finding patients. We know that most people don't know about clinical research, and few participate in clinical studies. New technologies can help us engage patients.

A second element is around end points. Many of the end points we have in clinical development are based on physician judgement, are relatively non-quantifiable or are dependent on patient diaries or patient-reported outcomes without strong standardization. We and others are looking hard at digital technologies, whether they are in watches or other sensors, to figure out whether we can quantify end points to give regulators more assurance that our drugs are having a substantial effect on a human disease. We are piloting this in chronic obstructive pulmonary disease (COPD), where we have put sensors into inhalers, and in conditions like heart failure, where we are working with sensors that track movement. I would say that we are in the exploratory phase with this. The real challenge now will be to convince regulators that these are validated end points that can supplant existing end points. I would expect to see this in the next 1-2 years.

Third, in terms of operationalization, there is no question that our processes for collecting and processing data are 20 years behind where they ought to be. If you think about the amount of paper that flows between clinical trial sites, and multiply that by the thousands of studies being run across the industry, there is a tremendous opportunity to use digital technologies. We've made commitments to invest much more in core systems that allow us to collect and analyse data efficiently, and then ultimately to get these data to regulators. 